\title{
On the existence and stability of solutions of a mixed general type of variational relation problems
}

\section{Zhe Yang*}

\section{"Correspondence:}

zheyang211@163.com

School of Economics, Shanghai

University of Finance and

Economics, Shanghai, 200433,

China

Key Laboratory of Mathematical Economics (SUFE), Ministry of

Education, Shanghai, 200433, China

\begin{abstract}
In this paper, we introduce a mixed general type of variational relation problems, and establish the existence theorem of solutions of mixed general types of variational relation problems. Moreover, we study the stability of a solution set of mixed general types of variational relation problems. We prove that most of mixed general types of variational relation problems (in the sense of Baire category) are essential and, for any mixed general type of variational relation problems, there exists at least one essential connected component of its solution set.
\end{abstract}

MSC: 49J53; 49J40

Keywords: mixed general types of variational relation problems; existence; generic stability; essential connected components

\section{Introduction}

It is well known that the equilibrium problems are unified models of several problems, namely optimization problems, saddle point problems, variational inequalities, fixed point problems, Nash equilibrium problems etc. Recently, Luc [1] introduced a more general model of equilibrium problems, which is called a variational relation problem (VR). The stability of the solution set of variational relation problems was studied in [2, 3]. Further studies of variational relation problems have been done (see [4-12]). Recently, Agarwal et $a l$. [13] presented a unified approach in studying the existence of solutions for two types of variational relation problems, which encompass several generalized equilibrium problems, variational inequalities and variational inclusions investigated in the recent literature. Balaj and Lin [14] established existence criteria for the solutions of two very general types of variational relation problems.

Motivated and inspired by research works mentioned above, we introduce mixed general types of variational relation problems, which is a mixed structure of two general types of variational relation problems in [14]. Moreover, we study the stability of a solution set of mixed general types of variational relation problems.

\section{Mixed general types of variational relation problems}

In [14], let $X, Y$ be convex sets in two Hausdorff topological vector spaces, $Z$ be a topological space, $S_{1}, S_{2}: X \rightrightarrows X, T: X \rightrightarrows Y, P: X \rightrightarrows Z$ be set-valued mappings with nonempty values, and $R(x, y, z)$ be a relation linking elements $x \in X, y \in Y$ and $z \in Z$. Balaj and Lin

C2014 Yang; licensee Springer. This is an Open Access article distributed under the terms of the Creative Commons Attribution License (http://creativecommons.org/licenses/by/2.0), which permits unrestricted use, distribution, and reproduction in any medium, provided the original work is properly cited. 
[14] established existence criteria for the solutions of the following variational relation problems:

(VRP1) Find $\left(x^{*}, y^{*}\right) \in X \times Y$ such that $x^{*} \in S_{1}\left(x^{*}\right), y^{*} \in T\left(x^{*}\right)$ and, $\forall u \in S_{2}\left(x^{*}\right), \exists z \in P\left(x^{*}\right)$ for which $R\left(u, y^{*}, z\right)$ holds.

(VRP2) Find $\left(x^{*}, y^{*}\right) \in X \times Y$ such that $x^{*} \in S_{1}\left(x^{*}\right), y^{*} \in T\left(x^{*}\right)$ and $R\left(u, y^{*}, z\right)$ holds $\forall u \in$ $S_{2}\left(x^{*}\right)$ and $\forall z \in P\left(x^{*}\right)$.

In this paper, we introduce mixed general types of variational relation problems. Let $X, Y$ be convex sets in two Hausdorff topological vector spaces, $Z$ be a topological space, $S: X \times Y \rightrightarrows X, T: X \times Y \rightrightarrows Y, H: X \times Y \rightrightarrows X, G: X \times Y \rightrightarrows Y, P: X \times Y \rightrightarrows Z$ be set-valued mappings with nonempty values, and $R(x, y, z), Q(y, x, z)$ be two relations linking elements $x \in X, y \in Y$ and $z \in Z$. A mixed general type of variational relation problems (MGVR) consists in finding $\left(x^{*}, y^{*}\right) \in X \times Y$ such that $x^{*} \in S\left(x^{*}, y^{*}\right), y^{*} \in T\left(x^{*}, y^{*}\right)$ and

$$
\begin{aligned}
& \forall u \in H\left(x^{*}, y^{*}\right), \quad \exists z \in P\left(x^{*}, y^{*}\right) \quad \text { s.t. } \quad R\left(u, y^{*}, z\right) \text { holds, } \\
& Q\left(v, x^{*}, z\right) \text { holds, } \quad \forall v \in G\left(x^{*}, y^{*}\right), \forall z \in P\left(x^{*}, y^{*}\right) .
\end{aligned}
$$

Remark 2.1 Balaj and Lin [14] established existence criteria for the solutions of two very general types of variational relation problems. The mixed general type of variational relation problems is a combination of (VRP1) and (VRP2), and (VRP1) and (VRP2) are some special cases of (MGVR).

\section{Theorem 2.1 Assume that}

(i) $X, Y, Z$ are three nonempty, compact and convex subsets of three Hausdorfflinear topological spaces;

(ii) $C=\{(x, y) \in X \times Y: x \in S(x, y)\}$ and $D=\{(x, y) \in X \times Y: y \in T(x, y)\}$ are closed in $X \times Y$

(iii) $P$ is continuous with nonempty compact values;

(iv) for any $(x, y) \in X \times Y$, co $H(x, y) \subset S(x, y)$, co $G(x, y) \subset T(x, y), H$, G have open fibers, and $R(x, \cdot, \cdot), Q(\cdot, y, \cdot)$ are closed;

(v) for any fixed $y \in Y$, any finite subset $\left\{u_{1}, \ldots, u_{n}\right\}$ of $X$ and any $x \in \operatorname{co}\left\{u_{1}, \ldots, u_{n}\right\}$, there are $i \in\{1, \ldots, n\}$ and $z \in P(x, y)$ such that $R\left(u_{i}, y, z\right)$ holds;

(vi) for any fixed $x \in X$, any finite subset $\left\{v_{1}, \ldots, v_{n}\right\}$ of $Y$ and any $y \in \operatorname{co}\left\{v_{1}, \ldots, v_{n}\right\}$, there is $i \in\{1, \ldots, n\}$ such that $Q\left(v_{i}, x, z\right)$ holds for any $z \in P(x, y)$.

Then (MGVR) has at least one solution.

Proof Define $A: X \times Y \rightrightarrows X$ and $B: X \times Y \rightrightarrows Y$ as follows:

$$
\begin{aligned}
& A(x, y)=\{u \in X: R(u, y, z) \text { does not hold } \forall z \in P(x, y)\}, \\
& B(x, y)=\{v \in Y: Q(v, x, z) \text { does not hold } \exists z \in P(x, y)\} .
\end{aligned}
$$

As $R(x, \cdot, \cdot), Q(\cdot, y, \cdot)$ are closed for any $(x, y) \in X \times Y$, and $P$ is continuous with nonempty compact values, by Propositions 3.1, 3.3 of [14], $A, B$ have open fibers.

Suppose that there exists $(x, y) \in X \times Y$ such that $x \in \operatorname{co} A(x, y)$, then there is a finite subset $\left\{u_{1}, \ldots, u_{n}\right\}$ of $A(x, y)$ such that $x \in \operatorname{co}\left\{u_{1}, \ldots, u_{n}\right\}$. By (v), there are $i_{0} \in\{1, \ldots, n\}$ and 
$z \in P(x, y)$ such that $R\left(u_{i_{0}}, y, z\right)$ holds, which contradicts the fact that $u_{i} \in A(x, y)$ for any $i \in\{1, \ldots, n\}$, i.e., $R\left(u_{i}, y, z\right)$ does not hold for any $z \in P(x, y)$ and any $i \in\{1, \ldots, n\}$. Hence $x \notin \operatorname{co} A(x, y)$ for any $(x, y) \in X \times Y$.

Suppose that there exists $(x, y) \in X \times Y$ such that $y \in \operatorname{co} B(x, y)$, then there is a finite subset $\left\{v_{1}, \ldots, v_{n}\right\}$ of $B(x, y)$ such that $y \in \operatorname{co}\left\{v_{1}, \ldots, v_{n}\right\}$. By (vi), there is $i_{0} \in\{1, \ldots, n\}$ such that $Q\left(v_{i_{0}}, x, z\right)$ holds, which contradicts the fact that $v_{i} \in B(x, y)$ for any $i \in\{1, \ldots, n\}$, i.e., there is $z \in P(x, y)$ such that $Q\left(v_{i}, x, z\right)$ does not hold for any $i \in\{1, \ldots, n\}$. Hence $y \notin \operatorname{co} B(x, y)$ for any $(x, y) \in X \times Y$.

Define $A^{\prime}: X \times Y \rightrightarrows X$ and $B^{\prime}: X \times Y \rightrightarrows Y$ as follows:

$$
\begin{aligned}
& A^{\prime}(x, y)= \begin{cases}A(x, y) \cap H(x, y) & \text { if }(x, y) \in C, \\
H(x, y) & \text { if }(x, y) \notin C,\end{cases} \\
& B^{\prime}(x, y)= \begin{cases}B(x, y) \cap G(x, y) & \text { if }(x, y) \in D, \\
G(x, y) & \text { if }(x, y) \notin D .\end{cases}
\end{aligned}
$$

For any $u \in X, A^{\prime-1}(u)=\left[H^{-1}(u) \cap A^{-1}(u)\right] \cup\left[((X \times Y) \backslash C) \cap H^{-1}(u)\right]$ is open in $X \times Y$. Similarly, $B^{\prime-1}(v)$ is open for any $v \in Y$. Hence, $A^{\prime-1}(u), B^{\prime-1}(v)$ are open for any $(u, v) \in X \times$ $Y$, and $x \notin \operatorname{co} A^{\prime}(x, y), y \notin \operatorname{co} B^{\prime}(x, y)$ for any $(x, y) \in X \times Y$. By Theorem 3 of [15], there exists $\left(x^{*}, y^{*}\right) \in X \times Y$ such that $A^{\prime}\left(x^{*}, y^{*}\right)=\emptyset$ and $B^{\prime}\left(x^{*}, y^{*}\right)=\emptyset$, which implies that $x^{*} \in S\left(x^{*}, y^{*}\right)$, $y^{*} \in T\left(x^{*}, y^{*}\right)$ and

$$
\begin{aligned}
& \forall u \in H\left(x^{*}, y^{*}\right), \quad \exists z \in P\left(x^{*}, y^{*}\right) \quad \text { s.t. } \quad R\left(u, y^{*}, z\right) \text { holds, } \\
& Q\left(v, x^{*}, z\right) \text { holds, } \quad \forall v \in G\left(x^{*}, y^{*}\right), \forall z \in P\left(x^{*}, y^{*}\right) .
\end{aligned}
$$

\section{Theorem 2.2 Assume that}

(i) $X, Y, Z$ are three nonempty, compact and convex subsets of three normed linear topological spaces;

(ii) $S, T$ are continuous with nonempty convex compact values;

(iii) $P$ is continuous with nonempty compact values;

(iv) $R(\cdot, \cdot, \cdot)$ and $Q(\cdot, \cdot, \cdot)$ are closed;

(v) for any fixed $y \in Y$, any finite subset $\left\{u_{1}, \ldots, u_{n}\right\}$ of $X$ and any $x \in \operatorname{co}\left\{u_{1}, \ldots, u_{n}\right\}$, there are $i \in\{1, \ldots, n\}$ and $z \in P(x, y)$ such that $R\left(u_{i}, y, z\right)$ holds;

(vi) for any fixed $x \in X$, any finite subset $\left\{v_{1}, \ldots, v_{n}\right\}$ of $Y$ and any $y \in \operatorname{co}\left\{v_{1}, \ldots, v_{n}\right\}$, there is $i \in\{1, \ldots, n\}$ such that $Q\left(v_{i}, x, z\right)$ holds for any $z \in P(x, y)$.

Then there exists $\left(x^{*}, y^{*}\right) \in X \times Y$ such that $x^{*} \in S\left(x^{*}, y^{*}\right), y^{*} \in T\left(x^{*}, y^{*}\right)$ and

$$
\begin{aligned}
& \forall u \in S\left(x^{*}, y^{*}\right), \quad \exists z \in P\left(x^{*}, y^{*}\right) \quad \text { s.t. } \quad R\left(u, y^{*}, z\right) \text { holds, } \\
& Q\left(v, x^{*}, z\right) \text { holds, } \quad \forall v \in T\left(x^{*}, y^{*}\right), \forall z \in P\left(x^{*}, y^{*}\right) .
\end{aligned}
$$

Proof For any $n$, define $S^{n}: X \times Y \rightrightarrows X, T^{n}: X \times Y \rightrightarrows Y, H^{n}: X \times Y \rightrightarrows X$ and $G^{n}: X \times Y \rightrightarrows$ $Y$ by

$$
\begin{aligned}
& S^{n}(x, y)=\left(S(x, y)+\operatorname{cl} V_{n}\right) \cap X, \quad T^{n}(x, y)=\left(T(x, y)+\operatorname{cl} V_{n}^{\prime}\right) \cap Y, \\
& H^{n}(x, y)=\left(S(x, y)+V_{n}\right) \cap X, \quad G^{n}(x, y)=\left(T(x, y)+V_{n}^{\prime}\right) \cap Y,
\end{aligned}
$$


where $V_{n}=\left\{x \in X:\|x\|<\frac{1}{n}\right\}, V_{n}^{\prime}=\left\{y \in Y:\|y\|<\frac{1}{n}\right\}$. Since $S, T$ are continuous, $H^{n}, G^{n}$ have open fibers, and $\operatorname{Graph}\left(S^{n}\right), \operatorname{Graph}\left(T^{n}\right)$ are closed in $X \times Y$, by Theorem 2.1, there exist $x^{n} \in S^{n}\left(x^{n}, y^{n}\right), y^{n} \in T^{n}\left(x^{n}, y^{n}\right)$ and

$$
\begin{array}{ll}
\forall u \in H^{n}\left(x^{n}, y^{n}\right), \quad \exists z \in P\left(x^{n}, y^{n}\right) \quad \text { s.t. } \quad R\left(u, y^{n}, z\right) \text { holds, } \\
Q\left(v, x^{n}, z\right) \text { holds, } \quad \forall v \in G^{n}\left(x^{n}, y^{n}\right), \forall z \in P\left(x^{n}, y^{n}\right) .
\end{array}
$$

Since $X, Y$ are nonempty and compact, without loss of generality, we assume that $\left(x^{n}, y^{n}\right) \rightarrow(x, y)$. Let $m>0$ be arbitrarily fixed and $n_{0}>0$ such that $V_{n_{0}}+V_{n_{0}}+\operatorname{cl} V_{n_{0}} \subset V_{m}$. Since $S$ is continuous and $\left(x^{n}, y^{n}\right) \rightarrow(x, y)$, there is $N_{1}>0$ such that $x-x^{n} \in V_{n_{0}}$ and $S\left(x^{n}, y^{n}\right) \subset S(x, y)+V_{n_{0}}$ for any $n>N_{1}$. Therefore, for any $n>\max \left\{N_{1}, n_{0}\right\}$,

$$
\begin{aligned}
x & =x-x^{n}+x^{n} \in x-x^{n}+S\left(x^{n}, y^{n}\right)+\operatorname{cl} V_{n} \\
& \subset V_{n_{0}}+S(x, y)+V_{n_{0}}+\operatorname{cl} V_{n_{0}} \subset S(x, y)+V_{m} .
\end{aligned}
$$

Hence $x \in \bigcap_{m>0}\left(S(x, y)+V_{m}\right)=\operatorname{cl} S(x, y)=S(x, y)$. Similarly, $y \in T(x, y)$.

Suppose that there exists $u \in S(x, y)$ such that $R(u, y, P(x, y))$ does not hold. Since $R(\cdot, \cdot, \cdot)$ is closed, there exists $k>0$ such that $R\left(u+V_{k}, y+V_{k}, P(x, y)+V_{k}\right)$ does not hold. Since $S$ is continuous, there exists a sequence $\left\{u^{n}\right\}$ convergent to $u$ with $u^{n} \in S\left(x^{n}, y^{n}\right) \subset S\left(x^{n}, y^{n}\right)+$ $V_{n} \subset H^{n}\left(x^{n}, y^{n}\right)$. Since $P$ is continuous, there exists $N_{0}>0$ such that, for any $n>N_{0}$, $u^{n} \in u+V_{k}, y^{n} \in y+V_{k}, P\left(x^{n}, y^{n}\right) \subset P(x, y)+V_{k}$, which implies that $u^{n} \in H^{n}\left(x^{n}, y^{n}\right)$ and $R\left(u^{n}, y^{n}, P\left(x^{n}, y^{n}\right)\right)$ does not hold. It is a contradiction.

Suppose that there exist $v \in T(x, y)$ and $z \in P(x, y)$ such that $Q(v, x, z)$ does not hold. Since $T, P$ are continuous, there exist two sequences $\left\{v^{n}\right\}$ and $\left\{z^{n}\right\}$ convergent to $v$ and $z$ with $v^{n} \in T\left(x^{n}, y^{n}\right) \subset T\left(x^{n}, y^{n}\right)+V_{n}^{\prime} \subset G^{n}\left(x^{n}, y^{n}\right)$ and $z^{n} \in P\left(x^{n}, y^{n}\right)$. As $Q(\cdot, \cdot, \cdot)$ is closed, there exists $N_{1}>0$ such that, for any $n>N_{1}, Q\left(v^{n}, x^{n}, z^{n}\right)$ does not hold, which is a contradiction. This completes the proof.

\section{Generic stability analysis}

Let $X, Y, Z$ be three nonempty, compact and convex subsets of three normed linear topological spaces. Denote by $\mathcal{M}$ the collection of all (MGVR) such that all conditions of Theorem 2.2 hold. For each $q \in \mathcal{M}$, denote by $F(q)$ the solution set of $q$. Thus, a correspondence $F: \mathcal{M} \rightrightarrows X \times Y$ is well defined. For each $q, q^{\prime} \in \mathcal{M}$, define the distance on $\mathcal{M}$ by

$$
\begin{aligned}
\rho\left(q, q^{\prime}\right)= & \sup _{(x, y) \in X \times Y} h_{X}\left(S(x, y), S^{\prime}(x, y)\right)+\sup _{(x, y) \in X \times Y} h_{Y}\left(T(x, y), T^{\prime}(x, y)\right) \\
& +\sup _{(x, y) \in X \times Y} h_{Z}\left(P(x, y), P^{\prime}(x, y)\right)+H\left(\operatorname{Gr}(R), \operatorname{Gr}\left(R^{\prime}\right)\right)+H\left(\operatorname{Gr}(Q), \operatorname{Gr}\left(Q^{\prime}\right)\right),
\end{aligned}
$$

where $\operatorname{Gr}(R)=\{(x, y, z) \in X \times Y \times Z: R(x, y, z)$ holds $\}, \operatorname{Gr}(Q)=\{(x, y, z) \in X \times Y \times Z$ : $Q(y, x, z)$ holds $\}, h_{X}\left(h_{Y}, h_{Z}\right)$ is the Hausdorff distance defined on $X(Y, Z)$, and $H$ is the Hausdorff distance defined on $X \times Y \times Z$.

Definition 3.1 Let $q \in \mathcal{M}$. An $(x, y) \in F(q)$ is said to be an essential point of $F(q)$ if, for any open neighborhood $N(x, y)$ of $(x, y)$ in $X \times Y$, there is a positive $\delta$ such that $N(x, y) \cap F\left(q^{\prime}\right) \neq \emptyset$ for any $q^{\prime} \in \mathcal{M}$ with $\rho\left(q, q^{\prime}\right)<\delta . q$ is said to be essential if each $(x, y) \in$ $F(q)$ is essential. 
Definition 3.2 Let $q \in \mathcal{M}$. A nonempty closed subset $e(q)$ of $F(q)$ is said to be an essential set of $F(q)$ if, for any open set $U, e(q) \subset U$, there is a positive $\delta$ such that $U \cap F\left(q^{\prime}\right) \neq \emptyset$ for any $q^{\prime} \in \mathcal{M}$ with $\rho\left(q, q^{\prime}\right)<\delta$.

Definition 3.3 Let $q \in \mathcal{M}$. An essential subset $m(q) \subset F(q)$ is said to be a minimal essential set of $F(q)$ if it is a minimal element of the family of essential sets in $F(q)$ ordered by set inclusion. A connected component $C(q)$ of $F(q)$ is said to be an essential component of $F(q)$ if $C(q)$ is essential.

Remark 3.1 (1) It is easy to see that the problem $q \in \mathcal{M}$ is essential if and only if the mapping $F: \mathcal{M} \rightrightarrows X \times Y$ is lower semicontinuous at $q$. (2) For two closed $e_{1}(q) \subset e_{2}(q) \subset$ $F(q)$, if $e_{1}(q)$ is essential, then $e_{2}(q)$ is also essential.

Lemma 3.1 (17.8 Lemma, 17.11 Closed Graph Theorem of [16]) (i) The image of a compact set under a compact-valued upper semicontinuous set-valued mapping is compact. (ii) A correspondence with compact Hausdorff range space is closed if and only if it is upper hemicontinuous and closed-valued.

Lemma 3.2 ([17]) If $X, Y$ are two metric spaces, $X$ is complete and $F: X \rightrightarrows Y$ is upper semicontinuous with nonempty compact values, then the set of points, where $F$ is lower semicontinuous, is a dense residual set in $X$.

Theorem $3.1(\mathcal{M}, \rho)$ is a complete metric space.

Proof Let $\left\{q^{n}\right\}_{n=1}^{\infty}$ be any Cauchy sequence in $\mathcal{M}$, then, for any $\varepsilon>0$, there is $N>0$ such that $\rho\left(q^{n}, q^{m}\right)<\varepsilon$ for any $n, m>N$, that is, for any $n, m>N$,

$$
\begin{aligned}
& \sup _{(x, y) \in X \times Y} h_{X}\left(S^{n}(x, y), S^{m}(x, y)\right)+\sup _{(x, y) \in X \times Y} h_{Y}\left(T^{n}(x, y), T^{m}(x, y)\right) \\
& \quad+\sup _{(x, y) \in X \times Y} h_{Z}\left(P^{n}(x, y), P^{m}(x, y)\right) \\
& \quad+H\left(\operatorname{Gr}\left(R^{n}\right), \operatorname{Gr}\left(R^{m}\right)\right)+H\left(\operatorname{Gr}\left(Q^{n}\right), \operatorname{Gr}\left(Q^{m}\right)\right) \leq \varepsilon .
\end{aligned}
$$

(1) Clearly, we consult Proposition 3.1 of [18]. There are $S: X \times Y \rightrightarrows X, T: X \times Y \rightrightarrows Y$ and $P: X \times Y \rightrightarrows Z$ such that $S, T$ are continuous with nonempty convex compact values, and $P$ is continuous with nonempty compact values.

(2) There exist two closed subsets $A, B$ of $X \times Y \times Z$ such that $\operatorname{Gr}\left(R^{n}\right) \rightarrow A$ and $\operatorname{Gr}\left(Q^{n}\right) \rightarrow B$. Denote $q=(S, T, P, R, Q)$, where

$$
R(x, y, z) \text { holds iff } \quad(x, y, z) \in A, \quad Q(y, x, v) \text { holds } \quad \text { iff } \quad(x, y, z) \in B .
$$

Clearly $R(\cdot, \cdot, \cdot)$ and $Q(\cdot, \cdot, \cdot)$ are closed.

(3) Suppose the existence of $y \in Y$, finite subset $\left\{u_{1}, \ldots, u_{n}\right\}$ of $X$ and $x \in \operatorname{co}\left\{u_{1}, \ldots, u_{n}\right\}$ such that $R\left(u_{i}, y, P(x, y)\right)$ does not hold for any $i \in\{1, \ldots, n\}$, which implies $\left(u_{i}, y, P(x, y)\right) \cap$ $\operatorname{Gr}(R)=\emptyset, \forall i \in\{1, \ldots, n\}$. Since $q^{m} \rightarrow q$ for enough large $m,\left(u_{i}, y, P^{m}(x, y)\right) \cap \operatorname{Gr}\left(R^{m}\right)=\emptyset$, $\forall i \in\{1, \ldots, n\}$, i.e., $R^{m}\left(u_{i}, y, P^{m}(x, y)\right)$ does not hold for any $i \in\{1, \ldots, n\}$, which is a contradiction. 
Suppose the existence of $x \in X$, finite subset $\left\{v_{1}, \ldots, v_{n}\right\}$ of $Y, y \in \operatorname{co}\left\{v_{1}, \ldots, v_{n}\right\}$ and $z \in P(x, y)$ such that $Q\left(v_{i}, x, z\right)$ does not hold for any $i \in\{1, \ldots, n\}$, which implies $\left(x, v_{i}, z\right) \notin$ $\operatorname{Gr}(Q), \forall i \in\{1, \ldots, n\}$. Since $q^{m} \rightarrow q$, there exists a sequence $\left\{z^{m}\right\}$ convergent to $z$ with $z^{m} \in P^{m}(x, y)$. Hence, for enough large $m,\left(x, v_{i}, z^{m}\right) \notin \operatorname{Gr}\left(Q^{m}\right), \forall i \in\{1, \ldots, n\}$, i.e., $Q^{m}\left(v_{i}, x, z^{m}\right)$ does not hold for any $i \in\{1, \ldots, n\}$, which is a contradiction. Hence $q \in \mathcal{M}$ and $(\mathcal{M}, \rho)$ is complete.

Theorem 3.2 The mapping $F: \mathcal{M} \rightrightarrows X \times Y$ is upper semicontinuous with nonempty compact values.

Proof The desired conclusion follows from Lemma 3.1 as soon as we show that $\operatorname{Graph}(F)$ is closed. Denote $q^{n}=\left(S^{n}, T^{n}, P^{n}, R^{n}, Q^{n}\right)$ and $q=(S, T, P, R, Q)$. Let $\left\{\left(q^{n}, x^{n}, y^{n}\right) \in \mathcal{M} \times X \times\right.$ $Y\}_{n=1}^{\infty}$ be a sequence converging to $(q, x, y)$ such that $\left(x^{n}, y^{n}\right) \in F\left(q^{n}\right)$ for any $n$. Then $x^{n} \in$ $S^{n}\left(x^{n}, y^{n}\right)$ and $y^{n} \in T^{n}\left(x^{n}, y^{n}\right)$,

$$
\begin{aligned}
& \forall u \in S^{n}\left(x^{n}, y^{n}\right), \quad \exists z \in P^{n}\left(x^{n}, y^{n}\right) \quad \text { s.t. } \quad R^{n}\left(u, y^{n}, z\right) \text { holds, } \\
& Q^{n}\left(v, x^{n}, z\right) \text { holds, } \quad \forall v \in T^{n}\left(x^{n}, y^{n}\right), \forall z \in P^{n}\left(x^{n}, y^{n}\right) .
\end{aligned}
$$

Clearly, $x \in S(x, y)$ and $y \in T(x, y)$.

Suppose the existence of $u \in S(x, y)$ such that $R(u, y, P(x, y))$ does not hold, then $(u, y, P(x, y)) \cap \operatorname{Gr}(R)=\emptyset$. Since $S, P$ are continuous, $q^{n} \rightarrow q$ and

$$
\begin{aligned}
& \quad h_{X}\left(S^{n}\left(x^{n}, y^{n}\right), S(x, y)\right) \\
& \quad \leq h_{X}\left(S^{n}\left(x^{n}, y^{n}\right), S\left(x^{n}, y^{n}\right)\right)+h_{X}\left(S\left(x^{n}, y^{n}\right), S(x, y)\right) \rightarrow 0, \\
& h_{Z}\left(P^{n}\left(x^{n}, y^{n}\right), P(x, y)\right) \\
& \quad \leq h_{Z}\left(P^{n}\left(x^{n}, y^{n}\right), P\left(x^{n}, y^{n}\right)\right)+h_{Z}\left(P\left(x^{n}, y^{n}\right), P(x, y)\right) \rightarrow 0,
\end{aligned}
$$

then $S^{n}\left(x^{n}, y^{n}\right) \rightarrow S(x, y)$ and $P^{n}\left(x^{n}, y^{n}\right) \rightarrow P(x, y)$. Thus, there exists a sequence $\left\{u^{n}\right\}$ convergent to $u$ with $u^{n} \in S^{n}\left(x^{n}, y^{n}\right)$ such that, for enough large $n,\left(u^{n}, y^{n}, P^{n}\left(x^{n}, y^{n}\right)\right) \cap \operatorname{Gr}\left(R^{n}\right)=$ $\emptyset$, i.e., $u^{n} \in S^{n}\left(x^{n}, y^{n}\right)$ and $R^{n}\left(u^{n}, y^{n}, P^{n}\left(x^{n}, y^{n}\right)\right)$ does not hold, which is a contradiction.

Suppose the existence of $v \in T(x, y)$ and $z \in P(x, y)$ such that $Q(v, x, z)$ does not hold, then $(x, v, z) \notin \operatorname{Gr}(Q)$. Similarly, $T^{n}\left(x^{n}, y^{n}\right) \rightarrow T(x, y)$ and $P^{n}\left(x^{n}, y^{n}\right) \rightarrow P(x, y)$. Thus, there exist two sequences $\left\{v^{n}\right\}$ and $\left\{z^{n}\right\}$ convergent to $u$ and $z$ with $v^{n} \in T^{n}\left(x^{n}, y^{n}\right)$ and $z^{n} \in P^{n}\left(x^{n}, y^{n}\right)$. Hence, for enough large $n,\left(x^{n}, v^{n}, z^{n}\right) \notin \operatorname{Gr}\left(Q^{n}\right)$, i.e., $v^{n} \in T^{n}\left(x^{n}, y^{n}\right), z^{n} \in P^{n}\left(x^{n}, y^{n}\right)$ and $Q^{n}\left(v^{n}, x^{n}, z^{n}\right)$ does not hold, which is a contradiction. Hence $(x, y) \in F(q)$.

Theorem 3.3 (i) There exists a dense residual subset $q$ of $\mathcal{M}$ such that $q$ is essential for each $q \in$ g. (ii) For any $q \in \mathcal{M}$, there exists at least one minimal essential subset of $F(q)$.

Proof The proofs are similar to those of Theorems 3.3 and 3.4 of [19]. Here, we do not repeat the process.

\section{Existence of essential connected components}

In this section, let $P_{0}: X \times Y \rightrightarrows Z$ be fixed. Assume that (i) $P_{0}$ is continuous with nonempty compact values; (ii) if $W_{1} \cap W_{2}=\emptyset, P_{0}\left(W_{1}\right) \cap P_{0}\left(W_{2}\right)=\emptyset$; (iii) $P_{0}^{-1}(z) \neq \emptyset$ for any $z \in Z$. Denote by $\mathcal{M}_{0}$ the collection of $\left(S, T, P_{0}, R, Q\right)$ mixed general types of variational relation 
problems such that all conditions of Theorem 2.2 hold. Clearly $\mathcal{M}_{0} \subset \mathcal{M}$. For convenience in the later presentation, for any subset $A$ of $X$, denote $A^{c}=\{x \in X: x \notin A\}$.

Lemma 4.1 ([20]) Let $C, D$ be two nonempty, convex and compact subsets of a linear normed space E. Then $h(C, \lambda C+\mu D) \leq h(C, D)$, where $h$ is the Hausdorff distance defined on $E$, and $\lambda, \mu \geq 0, \lambda+\mu=1$.

Lemma 4.2 ([21]) Let $(Y, \rho)$ be a metric space, $K_{1}$ and $K_{2}$ be two nonempty compact subsets of $Y, V_{1}$ and $V_{2}$ be two nonempty disjoint open subsets of $Y$. If $h\left(K_{1}, K_{2}\right)<\rho\left(V_{1}, V_{2}\right):=$ $\inf \left\{\rho(x, y) \mid x \in V_{1}, y \in V_{2}\right\}$, then

$$
h\left(K_{1},\left(K_{1} \backslash V_{2}\right) \cup\left(K_{2} \backslash V_{1}\right)\right) \leq h\left(K_{1}, K_{2}\right)
$$

where $h$ is the Hausdorff metric defined on $Y$.

Theorem 4.1 For any $q \in \mathcal{M}_{0}$, every minimal essential subset of $F(q)$ is connected.

Proof For each fixed $q \in \mathcal{M}_{0}$, let $m(q) \subset F(q)$ be a minimal essential subset of $F(q)$. If $m(q)$ is not connected, then there are two nonempty compact subsets $c_{1}(q), c_{2}(q)$ and two disjoint open subsets $V_{1}, V_{2}$ of $X \times Y$ such that $m(q)=c_{1}(q) \cup c_{2}(q)$ and $V_{1} \supset c_{1}(q), V_{2} \supset c_{2}(q)$. Since $m(q)$ is a minimal essential set of $F(q)$, neither $c_{1}(q)$ nor $c_{2}(q)$ is essential. There exist two open sets $O_{1} \supset c_{1}(q), O_{2} \supset c_{2}(q)$ such that, for any $\delta>0$, there exist $q^{1}, q^{2} \in \mathcal{M}_{0}$ with

$$
\rho\left(q, q^{1}\right)<\delta, \quad \rho\left(q, q^{2}\right)<\delta, \quad F\left(q^{1}\right) \cap O_{1}=\emptyset, \quad F\left(q^{2}\right) \cap O_{2}=\emptyset .
$$

Denote $W_{1}=V_{1} \cap O_{1}, W_{2}=V_{2} \cap O_{2}$, we know that $W_{1}, W_{2}$ are open, $W_{1} \supset c_{1}(q), W_{2} \supset c_{2}(q)$ and we may assume that $V_{1} \supset \bar{W}_{1}, V_{2} \supset \bar{W}_{2}$. Denote

$$
G_{1}=X \times Y \times\left(P_{0}\left(W_{2}^{c}\right)\right)^{c}, \quad G_{2}=X \times Y \times\left(P_{0}\left(W_{1}^{c}\right)\right)^{c} .
$$

Since $P_{0}$ is continuous with nonempty compact values, and $W_{1}^{c}, W_{2}^{c}$ are nonempty compact in $X \times Y$, by Lemma 3.1, $P_{0}\left(W_{1}^{c}\right), P_{0}\left(W_{2}^{c}\right)$ are nonempty compact in $Z$. Thus $G_{1}, G_{2}$ are open in $X \times Y \times Z$.

To prove by contraposition that $\left(P_{0}\left(W_{1}^{c}\right)\right)^{c} \cap\left(P_{0}\left(W_{2}^{c}\right)\right)^{c}=\emptyset$, suppose the existence of $z \in Z$ such that $z \in\left(P_{0}\left(W_{1}^{c}\right)\right)^{c} \cap\left(P_{0}\left(W_{2}^{c}\right)\right)^{c}$, which implies that $z \notin P_{0}\left(W_{1}^{c}\right)$ and $z \notin P_{0}\left(W_{2}^{c}\right)$, i.e., $W_{1}^{c} \cap P_{0}^{-1}(z)=\emptyset, W_{2}^{c} \cap P_{0}^{-1}(z)=\emptyset$. It follows that $P_{0}^{-1}(z) \subset W_{1}$ and $P_{0}^{-1}(z) \subset W_{2}$, which contradicts the fact that $W_{1} \cap W_{2}=\emptyset$.

Denote $\inf \left\{d(a, b) \mid a \in G_{1}, b \in G_{2}\right\}=\varepsilon>0$. Since $m(q)$ is essential, and $m(q) \subset\left(W_{1} \cup W_{2}\right)$, there exists $0<\delta^{*}<\varepsilon$ such that $F\left(q^{\prime}\right) \cap\left(W_{1} \cup W_{2}\right) \neq \emptyset$ for any $q^{\prime} \in \mathcal{M}_{0}$ with $\rho\left(q, q^{\prime}\right)<\delta^{*}$. Since $m(q)$ is a minimal essential set of $F(q)$, neither $c_{1}(q)$ nor $c_{2}(q)$ is essential. Thus, for $\frac{\delta^{*}}{32}>0$, there exist two $q^{1}, q^{2} \in \mathcal{M}_{0}$ such that

$$
F\left(q^{1}\right) \cap W_{1}=\emptyset, \quad F\left(q^{2}\right) \cap W_{2}=\emptyset, \quad \rho\left(q^{1}, q\right)<\frac{\delta^{*}}{32}, \quad \rho\left(q^{2}, q\right)<\frac{\delta^{*}}{32} .
$$

Thus $\rho\left(q^{1}, q^{2}\right)<\frac{\delta^{*}}{16}$. Next, define $q^{\prime}=\left(S^{\prime}, T^{\prime}, P_{0}, R^{\prime}, Q^{\prime}\right)$ as follows:

$$
\begin{aligned}
& S^{\prime}(x, y)=\lambda(x, y) S^{1}(x, y)+\mu(x, y) S^{2}(x, y), \\
& T^{\prime}(x, y)=\lambda(x, y) T^{1}(x, y)+\mu(x, y) T^{2}(x, y),
\end{aligned}
$$




$$
\begin{aligned}
& A=\left[\operatorname{Gr}\left(R^{1}\right) \backslash G_{2}\right] \cup\left[\operatorname{Gr}\left(R^{2}\right) \backslash G_{1}\right], \quad B=\left[\operatorname{Gr}\left(Q^{1}\right) \backslash G_{2}\right] \cup\left[\operatorname{Gr}\left(Q^{2}\right) \backslash G_{1}\right], \\
& R^{\prime}(u, y, z) \text { holds iff } \quad(u, y, z) \in A, \quad Q^{\prime}(v, x, z) \text { holds } \quad \text { iff } \quad(x, v, z) \in B,
\end{aligned}
$$

where

$$
\begin{array}{ll}
\lambda(x, y)=\frac{d\left((x, y), \bar{W}_{2}\right)}{d\left((x, y), \bar{W}_{1}\right)+d\left((x, y), \bar{W}_{2}\right)}, \quad \forall(x, y) \in X \times Y, \\
\mu(x, y)=\frac{d\left((x, y), \bar{W}_{1}\right)}{d\left((x, y), \bar{W}_{1}\right)+d\left((x, y), \bar{W}_{2}\right)}, \quad \forall(x, y) \in X \times Y .
\end{array}
$$

Easily, we check that (i) $S^{\prime}, T^{\prime}$ are continuous with nonempty compact convex values. (ii) Since $\operatorname{Gr}\left(R^{1}\right)$ and $\operatorname{Gr}\left(R^{2}\right)$ are closed in $X \times Y \times Z, A$ is closed in $X \times Y \times Z$, which implies that $R^{\prime}(\cdot, \cdot, \cdot)$ is closed. Similarly, $Q^{\prime}(\cdot, \cdot, \cdot)$ is closed. (iii) Suppose the existence of $y \in Y$, finite subset $\left\{u_{1}, \ldots, u_{n}\right\} \subset X$ and $x \in \operatorname{co}\left\{u_{1}, \ldots, u_{n}\right\}$ such that $R^{\prime}\left(u_{i}, y, P_{0}(x, y)\right)$ does not hold for any $i \in\{1, \ldots, n\}$, i.e., $\left(u_{i}, y, P_{0}(x, y)\right) \cap \operatorname{Gr}\left(R^{\prime}\right)=\emptyset, \forall i \in\{1, \ldots, n\}$. Since

$$
\operatorname{Gr}\left(R^{\prime}\right)=\left[\operatorname{Gr}\left(R^{1}\right) \backslash G_{2}\right] \cup\left[\operatorname{Gr}\left(R^{2}\right) \backslash G_{1}\right]
$$

and $W_{1} \cap W_{2}=\emptyset$, without loss of generality, we may assume that $(x, y) \in W_{1}^{c}$, which implies $P_{0}(x, y) \subset P_{0}\left(W_{1}^{c}\right)$. Since $\left(u_{i}, y, P_{0}(x, y)\right) \cap\left[\operatorname{Gr}\left(R^{2}\right) \backslash G_{1}\right]=\emptyset$ for all $i \in\{1, \ldots, n\}$, that is,

$$
\left(y, u_{i}, P_{0}(x, y)\right) \cap\left[\operatorname{Gr}\left(R^{2}\right) \cap\left(X \times Y \times P_{0}\left(W_{1}^{c}\right)\right)\right]=\emptyset, \quad \forall i \in\{1, \ldots, n\},
$$

then $\left(u_{i}, y, P_{0}(x, y)\right) \cap \operatorname{Gr}\left(R^{2}\right)=\emptyset, \forall i \in\{1, \ldots, n\}$, i.e., $R^{2}\left(u_{i}, y, P_{0}(x, y)\right)$ does not hold for any $i \in\{1, \ldots, n\}$, which is a contradiction.

(iv) Suppose the existence of $x \in X$, finite subset $\left\{v_{1}, \ldots, v_{n}\right\} \subset Y, y \in \operatorname{co}\left\{v_{1}, \ldots, v_{n}\right\}$ and $z \in P_{0}(x, y)$ such that $Q^{\prime}\left(v_{i}, x, z\right)$ does not hold for any $i \in\{1, \ldots, n\}$, i.e., $\left(x, v_{i}, z\right) \notin \operatorname{Gr}\left(Q^{\prime}\right)$, $\forall i \in\{1, \ldots, n\}$. Since

$$
\operatorname{Gr}\left(Q^{\prime}\right)=\left[\operatorname{Gr}\left(Q^{1}\right) \backslash G_{2}\right] \cup\left[\operatorname{Gr}\left(Q^{2}\right) \backslash G_{1}\right]
$$

and $W_{1} \cap W_{2}=\emptyset$, without loss of generality, we may assume that $(x, y) \in W_{1}^{c}$, which implies $z \in P_{0}(x, y) \subset P_{0}\left(W_{1}^{c}\right)$. Since $\left(x, v_{i}, z\right) \notin\left[\operatorname{Gr}\left(Q^{2}\right) \backslash G_{1}\right]$ for all $i \in\{1, \ldots, n\}$, that is, $\left(x, v_{i}, z\right) \notin$ $\left[\operatorname{Gr}\left(Q^{2}\right) \cap\left(X \times Y \times P_{0}\left(W_{1}^{c}\right)\right)\right]$ for all $i \in\{1, \ldots, n\}$, then

$$
\left(x, v_{i}, z\right) \notin \operatorname{Gr}\left(Q^{2}\right), \quad \forall i \in\{1, \ldots, n\},
$$

that is, there is $z \in P_{0}(x, y)$ such that $Q^{2}\left(v_{i}, x, z\right)$ does not hold for any $i \in\{1, \ldots, n\}$, which is a contradiction. Hence $q^{\prime} \in \mathcal{M}_{0}$.

(v) By Lemmas 4.1, 4.2,

$$
\begin{aligned}
\rho\left(q^{\prime}, q\right)= & \sup _{(x, y) \in X \times Y} h_{X}\left(S(x, y), S^{\prime}(x, y)\right)+\sup _{(x, y) \in X \times Y} h_{Y}\left(T(x, y), T^{\prime}(x, y)\right) \\
& +H\left(\operatorname{Gr}(R), \operatorname{Gr}\left(R^{\prime}\right)\right)+H^{\prime}\left(\operatorname{Gr}(Q), \operatorname{Gr}\left(Q^{\prime}\right)\right) \\
\leq & \sup _{(x, y) \in X \times Y} h_{X}\left(S(x, y), S^{1}(x, y)\right)+\sup _{(x, y) \in X \times Y} h_{X}\left(S^{1}(x, y), S^{\prime}(x, y)\right)
\end{aligned}
$$




$$
\begin{aligned}
& +\sup _{(x, y) \in X \times Y} h_{Y}\left(T(x, y), T^{1}(x, y)\right)+\sup _{(x, y) \in X \times Y} h_{Y}\left(T^{1}(x, y), T^{\prime}(x, y)\right) \\
& +H\left(\operatorname{Gr}(R), \operatorname{Gr}\left(R^{1}\right)\right)+H\left(\operatorname{Gr}\left(R^{1}\right), \operatorname{Gr}\left(R^{\prime}\right)\right) \\
& +H\left(\operatorname{Gr}(Q), \operatorname{Gr}\left(Q^{1}\right)\right)+H\left(\operatorname{Gr}\left(Q^{1}\right), \operatorname{Gr}\left(Q^{\prime}\right)\right) \\
& <\delta^{*} .
\end{aligned}
$$

Thus $q^{\prime} \in \mathcal{M}_{0}$ and $\rho\left(q^{\prime}, q\right)<\delta^{*}$.

Since $\left(F\left(q^{\prime}\right) \cap W_{1}\right) \cup\left(F\left(q^{\prime}\right) \cap W_{2}\right)=F\left(q^{\prime}\right) \cap\left(W_{1} \cup W_{2}\right) \neq \emptyset$, without loss of generality, we assume $F\left(q^{\prime}\right) \cap W_{1} \neq \emptyset$. Then there exists $(\bar{x}, \bar{y}) \in F\left(q^{\prime}\right) \cap W_{1}$ such that $(\bar{x}, \bar{y}) \in W_{1}, \bar{x} \in$ $S^{\prime}(\bar{x}, \bar{y}), \bar{y} \in T^{\prime}(\bar{x}, \bar{y})$, and

$$
\begin{aligned}
& \forall u \in S^{\prime}(\bar{x}, \bar{y}), \quad \exists z \in P_{0}(\bar{x}, \bar{y}) \quad \text { s.t. } \quad R^{\prime}(u, \bar{y}, z) \text { holds, } \\
& Q^{\prime}(v, \bar{x}, z) \text { holds, } \quad \forall v \in T^{\prime}(\bar{x}, \bar{y}), \forall z \in P_{0}(\bar{x}, \bar{y}) .
\end{aligned}
$$

It follows from $(\bar{x}, \bar{y}) \in W_{1}$ that $S^{\prime}(\bar{x}, \bar{y})=S_{1}(\bar{x}, \bar{y}), T^{\prime}(\bar{x}, \bar{y})=T_{1}(\bar{x}, \bar{y}), P_{0}(\bar{x}, \bar{y}) \subset P_{0}\left(W_{1}\right)$ and $P_{0}(\bar{x}, \bar{y}) \cap P_{0}\left(W_{1}^{c}\right)=\emptyset$. Therefore,

$$
\begin{aligned}
& \forall u \in S^{1}(\bar{x}, \bar{y}), \quad \exists z \in P_{0}(\bar{x}, \bar{y}) \quad \text { s.t. } \quad R^{1}(u, \bar{y}, z) \text { holds, } \\
& Q^{1}(v, \bar{x}, z) \text { holds, } \quad \forall v \in T^{1}(\bar{x}, \bar{y}), \forall z \in P_{0}(\bar{x}, \bar{y}) .
\end{aligned}
$$

Then $(\bar{x}, \bar{y}) \in F\left(q^{1}\right) \cap W_{1}$, which is a contradiction. This completes the proof.

Theorem 4.2 For any $q \in \mathcal{M}_{0}$, there exists at least one essential connected component of $F(q)$.

Proof By Theorem 4.1, there exists at least one connected minimal essential subset $m(q)$ of $F(q)$. Thus, there is a component $C$ of $F(q)$ such that $m(q) \subset C$. It is obvious that $C$ is essential by Remark 3.1(2). This completes the proof.

\section{Competing interests}

The author declares that they have no competing interests.

\section{Acknowledgements}

This research is supported the Chen Guang Project sponsored by the Shanghai Municipal Education Commission and Shanghai Education Development Foundation (no. 13CG35), and open project of Key Laboratory of Mathematical Economics (SUFE), Ministry of Education (no. 201309KF02).

Received: 11 April 2014 Accepted: 15 August 2014 Published: 02 Sep 2014

\section{References}

1. Luc, DT: An abstract problem in variational analysis. J. Optim. Theory Appl. 138, 65-76 (2008)

2. Khanh, PQ, Luc, DT: Stability of solutions in parametric variational relation problems. Set-Valued Var. Anal. 16(7-8), 1015-1035 (2008)

3. Pu, YJ, Yang, Z: Stability of solutions for variational relation problems with applications. Nonlinear Anal. 75, 1758-1767 (2012)

4. Lin, LJ, Wang, SY: Simultaneous variational relation problems and related applications. Comput. Math. Appl. 58, 1711-1721 (2009)

5. Balaj, M, Luc, DT: On mixed variational relation problems. Comput. Math. Appl. 60, 2712-2722 (2010)

6. Balaj, M, Lin, LJ: Equivalent forms of a generalized KKM theorem and their applications. Nonlinear Anal. 73, 673-682 (2010)

7. Balaj, M, Lin, LJ: Generalized variational relation problems with applications. J. Optim. Theory Appl. 148, 1-13 (2011)

8. Lin, L, Ansari, QH: Systems of quasi-variational relations with applications. Nonlinear Anal. 72, 1210-1220 (2010) 
9. Luc, DT, Sarabi, E, Soubeyran, A: Existence of solutions in variational relation problems without convexity. J. Math. Anal. Appl. 364, 544-555 (2010)

10. $\mathrm{Pu}, \mathrm{YJ}$, Yang, Z: Variational relation problem without the KKM property with applications. J. Math. Anal. Appl. 393, 256-264 (2012)

11. Yang, Z, Pu, YJ: Generalized Knaster-Kuratowski-Mazurkiewicz theorem without convex hull. J. Optim. Theory Appl. 154(1), 17-29 (2012)

12. Yang, Z, Pu, YJ: Existence and stability of solutions for maximal element theorem on Hadamard manifolds with applications. Nonlinear Anal. 75(2), 516-525 (2012)

13. Agarwal, RP, Balaj, M, O'Regan, D: A unifying approach to variational relation problems. J. Optim. Theory Appl. 155, 417-429 (2012)

14. Balaj, M, Lin, LJ: Existence criteria for the solutions of two types of variational relation problems. J. Optim. Theory Appl. $156,232-246(2013)$

15. Deguire, $P$, Tan, KK, Yuan, GXZ: The study of maximal elements, fixed points for $L_{s}$-majorized mapping and their applications to minimax and variational inequalities in the product topological spaces. Nonlinear Anal. 37, 933-951 (1999)

16. Aliprantis, CD, Border, KC: Infinite Dimensional Analysis: A Hitchhiker's Guide, 3rd edn. Springer, Berlin (2006)

17. Fort, MK Jr: A unified theory of semi-continuity. Duke Math. J. 16(2), 237-246 (1949)

18. Chen, JC, Gong, XH: The stability of set of solutions for symmetric vector quasi-equilibrium problems. J. Optim. Theory Appl. 136, 359-374 (2008)

19. Yang, Z: On existence and essential stability of solutions of symmetric variational relation problems. J. Inequal. Appl. 2014, 5 (2014)

20. Yu, J, Luo, Q: On essential components of the solution set of generalized games. J. Math. Anal. Appl. 230, 303-310 (1999)

21. Yu, J, Zhou, YH: A Hausdorff metric inequality with applications to the existence of essential components. Nonlinear Anal. 69, 1851-1855 (2008)

10.1186/1029-242X-2014-337

Cite this article as: Yang: On the existence and stability of solutions of a mixed general type of variational relation problems. Journal of Inequalities and Applications 2014, 2014:337

\section{Submit your manuscript to a SpringerOpen ${ }^{\circ}$ journal and benefit from:}

- Convenient online submission

Rigorous peer review

- Immediate publication on acceptance

- Open access: articles freely available online

- High visibility within the field

- Retaining the copyright to your article 